\title{
Uma Análise Tecnico-Econômica da Viabilidade da Prestação de Telefonia Rural no Brasil
}

\author{
Eduardo Jorge Brito Rodrigues
}

\begin{abstract}
Resumo- O provimento de telecomunicações rurais em países em desenvolvimento é um desafio para prestadoras e órgãos governamentais. Em 2008, a Anatel ressaltou a importância da criação de ofertas inovadoras de telecomunicações em áreas rurais. Apresentamos uma análise tecnico-econômica da prestação auto-sustentável de telefonia rural sob o espectro $450 \mathrm{MHz}$. Na analise, Capex, Opex, estimativas de mercado e das tarifas são combinados a fim de extrair conclusões acerca da rentabilidade do negócio. Os resultados obtidos no estudo são baseados em indicadores econômicos e podem auxiliar projetistas de rede e investidores na tomada de decisões.
\end{abstract}

Palavras-Chave - telefonia rural, Capex, Opex, WLL, $450 \mathrm{MHz}$.

Abstract- The provision of rural telecommunications in developing countries is a challenge for providers and government agencies. In 2008, Anatel emphasized the importance of creating innovative offerings of telecommunications services in rural areas. We present a technical-economic analysis of the provision of profitable rural telephony over $450 \mathrm{MHz}$ spectrum. In the analysis, Capex, Opex, market forecasts and tariffs are combined to draw conclusions about the profitability of the business. The results obtained in the case study are based on economic indicators and can help network designers and investors in making decisions.

Key-words - rural telephony, Capex, Opex, WLL, $450 \mathrm{MHz}$.

\section{INTRODUÇão}

Os desafios na implantação das telecomunicações em países em desenvolvimento vem sendo analisados há muitos anos. No caso da América Latina, diferenças nas condições políticas, climáticas e geográficas, dentre outros fatores, ocasionaram desenvolvimento diferenciado em cada país [1]. No Brasil, a Agência Nacional de Telecomunicações - Anatel, vinculada ao Ministério das Comunicações, tem função de órgão regulador do setor. Uma de suas missões é implementar a política nacional de telecomunicações. No tocante aos serviços de telecomunicações rurais, o Plano Geral para Atualização da Regulamentação de Telecomunicações no Brasil (PGR), aprovado pela Resolução n. ${ }^{\circ}$ 516/2008, ressaltou a importância da criação de oferta de serviços a preços módicos em áreas rurais, considerando novas maneiras de amortização de investimentos e novas formas de prestação do serviço.

Eduardo Jorge Brito Rodrigues, Agência Nacional de Telecomunicações Anatel - DF e Programa de Pós-Graduação em Engenharia Elétrica e Computação, Universidade Federal do Rio Grande do Norte - UFRN, Brasil, E-mail: eduardo_telecom@yahoo.com.br.
Devido às grandes diversidades sociais, econômicas e geográficas, planejar serviços de telecomunicações viáveis no Brasil torna-se um desafio diário tanto para as prestadoras dos serviços quanto para os organismos governamentais. Desde a Lei Geral de Telecomunicações (Lei n. ${ }^{\circ}$ 9.472/97) até os dias atuais, observa-se a completa universalização do acesso à telefonia fixa em áreas urbanas, visto que o Serviço Telefônico Fixo Comutado (STFC) está disponível em 100\% (5.564) dos Municípios, que corresponde a 36.406 localidades e atende população superior a 158 milhões de habitantes. Há provimento de acessos individuais em todas as localidades com pelo menos 300 habitantes e há Terminais de Uso Público (TUP) em localidades com pelo menos 100 habitantes, incluindo aldeias indígenas, localidades isoladas e fronteiriças. De forma similar, o país chegou a 153 milhões de assinantes do Serviço Móvel Pessoal (SMP), onde $81,61 \%$ são pré-pagos, e $18,39 \%$ pós-pagos [2]. No entanto, grande parte da cobertura das redes do SMP em tecnologia $3 \mathrm{G}$ concentra-se nas áreas urbanas.

Neste trabalho é apresentada uma análise tecnicoeconômica da viabilidade da prestação auto-sustentável do STFC, por meio de acessos fixos sem fio WLL (Wireless Local Loop), baseado na tecnologia CDMA (Code Division Multiple Access) sob o espectro de $450 \mathrm{MHz}$. Na análise, um estudo de caso da prestação do serviço foi elaborado para regiões rurais do Estado do Rio Grande do Sul (RS). O trabalho é organizado da seguinte forma: na seção II, é feito um panorama do atendimento rural no Brasil; Na seção III, são apresentadas algumas das alternativas tecnológicas possíveis; Na seção IV, experiências internacionais no atendimento rural são mostradas; Na seção V, o modelo proposto é descrito; Na seção VI, são mostrados os resultados obtidos e, por fim, na seção VII, são apresentadas as conclusões do estudo.

\section{ATENDIMENTO RURAL NO BRASIL}

Atualmente, a inexistência de serviços de telecomunicações em regiões rurais de todo o mundo é um problema mais de ordem econômica do que tecnológica, especialmente nas regiões rurais de países em desenvolvimento, os quais tem baixa penetração dos serviços e população espaça. $\mathrm{O}$ acesso à comunicação instantânea é fundamental para diversas atividades como agricultura, indústria, pecuária, educação e serviços de saúde. A conectividade entre áreas rurais e urbanas pode superar as barreiras da distância, as quais entravam o desenvolvimento rural [3]. No Brasil, apesar dos esforços para se ampliar os serviços de telecomunicações nas regiões mais afastadas das áreas urbanas, observa-se que ainda não existe uma alternativa consolidada para o atendimento rural com o 
STFC. Historicamente, uma das alternativas mais difundidas no país é o serviço Ruralcel, legado do Sistema Telebrás, onde há um compartilhamento remunerado da infraestrutura de acesso do SMP para atendimento a usuários situados, conforme a regulamentação, fora da Área de tarifa Básica (FATB) do STFC. Este usuário rural arca com as tarifas do serviço em área urbana, acrescidas de um preço pelos meios adicionais (airtime) cobrados por minuto, Figura 1. Sua oferta comercial tem se tornado pouco interessante tanto para os mais de 45 mil usuários ativos quanto para as Concessionárias do STFC. Tecnicamente ultrapassada, a tecnologia foi adotada para comunicações rurais em vários países como Índia, Malásia e Arábia Saudita [4].

\section{Alternativas TECNOLÓGICAS}

As recomendações feitas pela Anatel no PGR em 2008, acerca da criação de novas formas de prestação e oferta de serviços nas áreas rurais, podem ser contempladas com base no uso de tecnologias inovadoras ou, ainda, por meio de novos modelos de negócios utilizando tecnologias já conhecidas. A disponibilização de acessos individuais nas redes de telecomunicações em zonas rurais, por meio de cabos metálicos, fibras ópticas ou satélites, tem se mostrado pouco atrativa do ponto de vista econômico. Além disso, a maioria das aplicações requeridas nessas regiões não exige grande capacidade de transmissão. Tecnologias emergentes como o WiMAX (Worldwide Interoperability for Microwave Access) e o PLC (Power Line Communications), podem futuramente ganhar escala e se tornar atrativas. Prestado por meio das redes de energia elétrica, o PLC já tem regulamentação específica no Brasil. Testes realizados em 2007 demonstraram que a implementação de técnicas de mitigação de interferências e controle de potência de saída podem oferecer a proteção necessária na garantia da coexistência do PLC com outros sistemas que compartilham a mesma faixa de frequências [5]. O WiMAX tem sido apontado como solução para a massificação do acesso banda larga fixa e móvel, sendo alternativa à soluções como o DSL (Digital Subscriber Line) e o CableModem. No entanto, a falta de equipamentos de rede e de usuários em fabricados escala, assim como as características das faixas 2.6 e $3.5 \mathrm{GHz}$ não favorecem a aplicação desses sistemas em ambientes com população espaça [6].

Neste trabalho analisaremos a viabilidade do atendimento rural por meio da prestação do STFC com rede WLL e tecnologia CDMA2000, na faixa de 450-470 $\mathrm{MHz}$. Em rede WLL, os assinantes são conectados ao STFC por meio de enlaces sem fios, o que torna este tipo de rede uma alternativa bastante viável para a conectividade em áreas rurais.

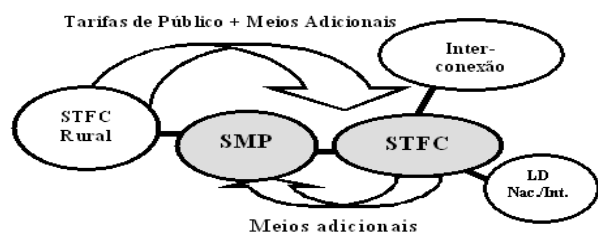

Figura 1. Serviço Ruralcel.
O WLL oferece implantação fácil, custos de instalação e manutenção reduzidos e independe de tecnologia, podendo ser utilizados, dentre vários, os padrões GSM (Global System for Mobile Communications), OFDMA (Orthogonal Frequency Division Multiple Access) ou CDMA [7]. A inexistência de processos como handoff e roaming, além da restrição da mobilidade dos usuários no STFC com WLL, possibilitam menores custos operacionais em relação às redes do SMP. Com mobilidade restrita, a rede WLL funciona da mesma forma que uma rede com fios, por tanto não há competição entre as prestadoras do STFC e do SMP. Além disso, o Brasil é um dos países onde há experiências bem sucedidas em restrição de mobilidade em redes do STFC com WLL [8].

\section{CONTEXTO INTERNACIONAL}

Na Recomendação CCP.II/Rec. 10 (V-05) sobre o uso da faixa de 450-470 $\mathrm{MHz}$, para serviços fixos e móveis em áreas de baixa densidade demográfica, a CITEL (Comissão Interamericana de Telecomunicações) orientou os países das Américas a considerar, onde o espectro possa ser utilizado, uso dessa faixa para telecomunicações em zonas mais remotas, e realizar ações técnicas e regulatórias de modo que os sistemas novos e os antigos possam coexistir sem interferências. $\mathrm{Na}$ Resolução n. ${ }^{\circ} 224$ (Rev. CMR-07) a União Internacional de Telecomunicações (UIT) identificou as faixas de 450$470 \mathrm{MHz}$ para sistemas IMT-2000 (International Mobile Telecommunications - 2000). A UIT reconhece que esta faixa pode contribuir para a redução das lacunas existentes entre as zonas pouco povoadas e as zonas com grande densidade demográfica. Além disso, a identificação de uma banda para o IMT-2000 não exclui a utilização desta por outros serviços e aplicações que lhes sejam atribuídas.

A escolha do CDMA450 neste trabalho foi baseada em características técnicas e nas experiências internacionais bem sucedidas no atendimento a áreas rurais. O CDMA450 é a combinação do CDMA2000 com o espectro $450 \mathrm{MHz}$, que apresenta características de propagação superiores às encontradas nas demais faixas destinadas ao IMT-2000, e possibilita maior alcance das Estações Base (BS) com consequente redução do Capex (Capital Expenditures) e do Opex (Operational Expenditures). O CDMA2000 é uma tecnologia estabelecida, com 100 operações comerciais em 52 países e 14 países com instalações programadas para 2010 [9]. Equipamentos de rede e terminais de usuários são desenvolvidos em escala global, por fabricantes como Qualcomm, Huawei, ZTE, Alcatel-Lucent e Nortel. Uma BS CDMA450 pode ter raio de cobertura de até $50 \mathrm{~km}$. Comparando com os raios médios nas faixas $800 \mathrm{MHz}$, a cobertura CDMA450 chega a ser $41 \%$ superior. Se comparada com o raio médio em $1900 \mathrm{MHz}$, a cobertura é 73\% maior. Além disso, o padrão apresenta desempenho satisfatório tanto em áreas urbanas quanto em áreas rurais, com eficiência espectral e capacidade de transmissão elevadas, alta qualidade de voz, mitigação do efeito multipercursos, controle de potencia sofisticado, serviços de dados e de voz integrados, terminais inteligentes de usuários e evolução tecnológica garantida [10], [11]. Na Europa, a Zapp Telemobil lançou a primeira operação comercial de telefonia fixa com CDMA450 na Romênia. Em menos de 5 anos, a operadora já possuía mais 
de 7 milhões de assinantes. Existe operação com CDMA450 em outros países como Portugal, Rússia, Dinamarca, e República Tcheca. Na Ásia, o Ministério de TI e Telecom do Paquistão disponibilizou licenças nos espectros 450 e $479 \mathrm{MHz}$ para prestação de WLL em 14 regiões, visando expandir a teledensidade em milhares de vilas e cidades do país. Os operadores optaram pelo CDMA450 com WLL, e operam com mobilidade restrita, determinada pelo regulador local [7]. China, Índia, Malásia e Arábia Saudita são alguns dos 20 países que possuem oferta comercial do CDMA450 na região. Nas Américas, a Telmex (México) implantou o CDMA450 para prestação de telefonia fixa e conexão à Internet, visando atender 15 mil localidades, com suporte financeiro do Fundo de Cobertura Social de Telecomunicações. Em algumas BS, é possível realizar chamadas com terminais fixos situados a $98 \mathrm{~km}$ de distância [9]. Na Argentina, a Cotecal possui oferta comercial de telefonia fixa e acesso à Internet com CDMA450. Além dela, há outras 4 operadoras usando a faixa $450 \mathrm{MHz}$. Equador e Peru também tem oferta comercial de serviços telefônicos fixos e móveis em áreas urbanas e rurais. No Brasil, o Ministério das Comunicações publicou a Portaria n. ${ }^{\circ} 431$, de 23 de julho de 2009, instituindo o Programa Nacional de Telecomunicações Rurais, privilegiando o uso do espectro na faixa de $450-470 \mathrm{MHz}$ para atendimento às populações rurais e com previsão de início do atendimento no ano de 2010 [2].

\section{DESCRIÇÃO DO MODELO}

\section{A. Cenários e público alvo}

Estima-se em 30 milhões de habitantes, distribuídos em 8 milhões de domicílios, a população rural no Brasil. Desses, aproximadamente $20 \%$ possuem renda mensal maior que 3 salários mínimos. Em muitas localidades, este mercado com potencial de consumo não possui oferta de serviços de telecomunicações [12]. O RS foi escolhido para esta análise pois além de ter a maior base de clientes do Ruralcel, possui grandes regiões cuja economia depende do agronegócio. Do total de 496 municípios do RS, apenas 18 tem população maior que 100 mil habitantes. Nesses, há baixa teledensidade e pouca oferta de serviços em áreas FATB. Na Tabela I, são ilustrados os dados referentes à área e à população do RS, onde a média de habitantes por domicílio é 3,51 [13]. Na Tabela II, é apresentada uma estimativa da população rural do RS nos 5 anos considerados na análise. Neste trabalho são estabelecidos três cenários de operação: No Cenário 1, uma projeção do quantitativo de domicílios rurais sem telefone (fixo ou móvel) no RS foi considerada como sendo o público alvo do serviço [12]. Este Cenário traduz a perspectiva pessimista do modelo; O Cenário 2, traduz a perspectiva mais realista deste trabalho. Nele, o mesmo público do Cenário 1 foi considerado, sendo a este acrescentado $30 \%$ dos usuários que já possuem telefones (Ruralcel ou SMP, e.g.) e residem em áreas rurais; O Cenário 3 , traduz a perspectiva mais otimista do plano. Neste caso, $50 \%$ dos domicílios rurais das projeções são considerados como sendo o público alvo. De acordo com a renda mensal, os domicílios foram classificados como potenciais consumidores dos serviços pré ou pós-pagos. Os quantitativos de usuários de cada cenário são apresentados na Figura 2. A penetração do serviço considerada no ciclo de 5 anos de operação é representada na Tabela III.
TABELA I. QuANTITATIVOS UtILIZADOS NA ANÁLISE.

\begin{tabular}{|c|c|c|c|}
\hline Municípios & Pop. Total & Pop. Rural & Área $\left(\mathbf{k m}^{\mathbf{}}\right)$ \\
\hline Pop. total RS & 10.187 .798 & 1.869 .814 & 258.619 \\
\hline Pop. $<100$ mil & 5.490 .023 & 1.659 .827 & 235.981 \\
\hline
\end{tabular}

TABELA II. PopulaÇão Estimada RS (MILHÕES)

\begin{tabular}{|c|c|c|c|c|c|}
\hline Ano & $\mathbf{2 0 1 2}$ & $\mathbf{2 0 1 3}$ & $\mathbf{2 0 1 4}$ & $\mathbf{2 0 1 5}$ & $\mathbf{2 0 1 6}$ \\
\hline Total & 11,07 & 11,12 & 11,16 & 11,21 & 11,25 \\
\hline Rural & 1,74 & 1,75 & 1,76 & 1,77 & 1,77 \\
\hline
\end{tabular}

TABela III. PenetraÇão do SERviço na ANÁlise .

\begin{tabular}{|c|c|c|c|c|c|}
\hline Ano & $\mathbf{2 0 1 2}$ & $\mathbf{2 0 1 3}$ & $\mathbf{2 0 1 4}$ & $\mathbf{2 0 1 5}$ & $\mathbf{2 0 1 6}$ \\
\hline Pré-pago (\%) & 30 & 35 & 40 & 45 & 50 \\
\hline Pós-pago (\%) & 40 & 45 & 50 & 55 & 60 \\
\hline Total (\%) & 33 & 38 & 43 & 48 & 53 \\
\hline
\end{tabular}

\section{B. Considerações econômicas}

O modelo de prestação desenhado contempla a criação do Valor de Uso da rede Rural (VU-R), que serve para remunerar a prestadora do STFC em áreas rurais pelas chamadas terminadas em sua rede WLL e é coerente com os modelos de remuneração vigentes no Brasil como, por exemplo, o modelo de remuneração do SMP. No estudo, uma perspectiva de redução gradual da VU-R foi considerado. Domicílios com renda menor que 3 salários mínimos foram classificados como potenciais usuários pré-pago e os com renda maior que 3 salários mínimos como potenciais usuários pós-pago. A cesta de produtos pós-pago é exibida na Tabela IV. Com base nesses parâmetros, foi possível determinar o valor da receita mensal por usuário (ARPU). A cesta pré-pago é detalhada na Tabela V. A receita global é dada pela soma da ARPU com a receita gerada pelo usuário com VU-R. No modelo, todos os usuários originam chamadas Locais e Longa Distância Nacional ou Internacional (LD). A Assinatura Básica, os valores dos minutos Locais e LD são reajustados em função do Índice de Serviços de Telecomunicações (IST) e do Fator de Produtividade (Fator $\mathrm{X}$ ), segundo as normas de tarifação da Anatel [2]. O valor do reajuste considerado foi $2 \%$ a.a. Todos os valores estimados são líquidos de impostos.

A análise tecnico-econômica foi realizada com base em índices financeiros como o Valor Presente Líquido (VPL). Assim, utilizamos uma estimativa do WACC (Weighted Average Cost of Capital) para o STFC, modelado pela Fundação Getúlio Vargas, com o objetivo de trazer a valores presentes as projeções dos resultados do projeto. O cálculo consiste na subtração de todas as despesas da operação do total de receitas auferidas, ambas projetadas para cada ano simulado. Para simplificação do modelo, foi utilizado o mesmo WACC (12\%) para todo período [2]. O VPL representa, no presente, o volume de capital esperado durante um determinado período de tempo. Se o VPL for positivo, o projeto é aceitável i.e., há bons indicadores da rentabilidade dos investimentos tendo em conta o tempo e o custo de oportunidade do capital, expresso pelo WACC [14]. 


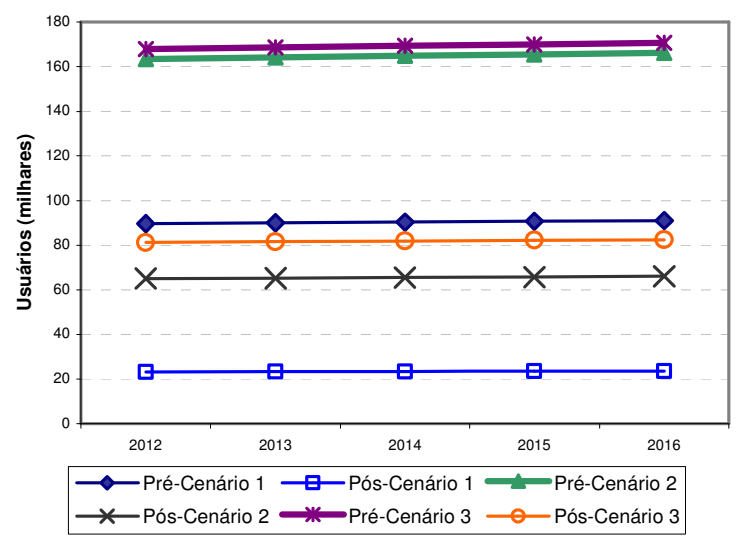

Figura 2. Quantitativos de usuários.

TABELA IV. CESTA DE PRODUTOS PÓS-PAGO.

\begin{tabular}{|c|c|}
\hline Assinatura básica $(\mathrm{R} \$)$ & A \\
\hline Franquia de minutos $(\mathrm{min})$ & $\mathrm{B}$ \\
\hline VU-R $(\mathrm{R} \$)$ & $\mathrm{C}$ \\
\hline Minutos Locais originados & $\mathrm{D}$ \\
\hline Minutos LD originados & $\mathrm{E}$ \\
\hline Minutos recebidos & $\mathrm{F}$ \\
\hline Minuto Local $(\mathrm{R} \$)$ & $\mathrm{G}$ \\
\hline Minuto LD $(\mathrm{R} \$)$ & $\mathrm{H}$ \\
\hline ARPU $(\mathrm{R} \$)$ & $\mathrm{i}=\mathrm{a}+(\mathrm{d}-\mathrm{b}) \cdot \mathrm{g}+(\mathrm{e} \cdot \mathrm{h})$ \\
\hline Minuto entrante $(\mathrm{VU}-\mathrm{R})$ & $\mathrm{j}=\mathrm{c} \cdot \mathrm{f}$ \\
\hline Receita Global $(\mathbf{R} \$)$ & $\mathbf{k}=\mathbf{i}+\mathbf{j}$ \\
\hline
\end{tabular}

TABELA V. CESTA DE PRODUTOS PRÉ-PAGO.

\begin{tabular}{|c|c|}
\hline VU-R $(\mathrm{R} \$)$ & $\mathrm{a}$ \\
\hline Minutos Locais originados & $\mathrm{b}$ \\
\hline Minutos LD originados & $\mathrm{c}$ \\
\hline Minutos recebidos & $\mathrm{d}$ \\
\hline Minuto Local $(\mathrm{R} \$)$ & $\mathrm{e}$ \\
\hline Minuto LD $(\mathrm{R} \$)$ & $\mathrm{f}$ \\
\hline ARPU $(\mathrm{R} \$)$ & $\mathrm{g}=(\mathrm{b} \cdot \mathrm{e})+(\mathrm{c} \cdot \mathrm{f})$ \\
\hline Minuto entrante $(\mathrm{VU}-\mathrm{R})(\mathrm{R} \$)$ & $\mathrm{h}=(\mathrm{a} \cdot \mathrm{d})$ \\
\hline Receita Global $(\mathbf{R} \$)$ & $\mathbf{i}=\mathbf{g}+\mathbf{h}$ \\
\hline
\end{tabular}

\section{Considerações Técnicas}

O CDMA450 consiste na operação do CDMA2000 sob o espectro $450 \mathrm{MHz}$. No CDMA450 1xRTT, um par de frequências de 1,25 $\mathrm{MHz}$ é requerido para suportar tráfego de voz e dados em até $153 \mathrm{kbps}$ por enlace. O sistema oferece mais de 35 canais de voz por setor por carrier, o que o torna um dos mais eficientes em uso do espectro da atualidade [10].

As versões mais avançadas do padrão, e.g. as revisões A, B e C do 1xEVDO (Evolution Data Optimized), são requeridas para fornecimento de serviços banda larga, por isso não foram consideradas neste trabalho. $\mathrm{Na}$ análise, consideramos um raio da BS CDMA450 de $25 \mathrm{~km}$, intermediário entre os de [15] e [11], e que propicia uma área de cobertura de $1.625 \mathrm{~km}^{2}$ com sistemas irradiantes omnidirecionais. Considera-se que cada uma das BS tem, em princípio, um par de portadoras de 1,25 $\mathrm{MHz}$ operando em modo FDD (Frequency Division Duplex).

Um fator de cobertura de $80 \%$ foi adotado e as áreas inóspitas, como topos de montanhas, rios e vales, foram desconsideradas. Além disso, a construção de infra-estrutura de core de rede, backbone, energia, banco de baterias, etc. foi considerada padrão para todos os cenários. Não foram estimados custos adicionais com o incremento de canalização nas BS com o crescimento da demanda, assim como não foi considerada a otimização desses recursos em caso de baixa utilização. Estas particularidades devem ser acompanhadas pelo operador, no primeiro ciclo de operação e manutenção, inerente à conclusão de um projeto de redes sem fios baseado na tecnologia CDMA.

\section{Capex e Opex}

Neste trabalho, chamamos os investimentos que compõem o Capex de: SITE_BS (custos totais das BS, incluindo equipamentos transceptores de RF, shelter, torre, cabos, antenas de RF e conectores); INFRA_BS (custos com infraestrutura e alvenaria); TX_BS (equipamentos do backbone como rádios, modems, antenas e guias de onda, por BS); ENE_BS (equipamentos energia como banco de baterias, postes e transformadores, por BS); INST_BS (instalação dos equipamentos, por BS); MS_AQ (custo aquisição de equipamentos de usuário); ANA_TF (tributos devidos à Anatel como taxas de fiscalização e de instalação); CORE (custos com equipamentos componentes da CCC, com instalação).

O Opex é composto por: O\&MR (operação e manutenção de rede, que inclui custos com funcionários, licenças de software, manutenção e reparo de equipamentos); V\&M (vendas e marketing, que inclui os custos com captação de clientes, vendas e propagandas); SAC (serviços ao cliente, onde consideramos os custos com callcenter, cobranças e auxílio à lista telefônica); TI\&INFRA (Suporte de informática, infraestrutura e interconexão de redes, contemplando os custos com informática, infraestrutura, aluguel de rede e terrenos, energia, interconexão com outras operadoras e terminação de chamadas).

O Capex e o Opex são apresentados nas Figuras 3 e 4. Mais de $70 \%$ dos investimentos concentram-se na rede de acesso WLL (SITE_BS), enquanto que os custos com o núcleo da rede (CORE) representam $4,48 \%$ do total. A construção do backbone (TX_BS) representa 13,40\% dos investimentos requeridos. A maior parcela do Opex está atrelada à $\mathrm{O} \& M R$ $(60,34 \%)$. Os custos com SAC e V\&M em áreas rurais são baixos devido à demanda ser reprimida e à falta de competição no segmento. Custos com TI\&INFRA representam $32,61 \%$ do Opex. Durante os primeiros anos, o Opex aumenta, principalmente, por causa do crescimento da base de usuários. Com a operação estabilizada, i.e., pequenos incrementos na base de clientes e domínio das tecnologias, há possibilidade de redução dos custos de O\&MR e V\&M. 


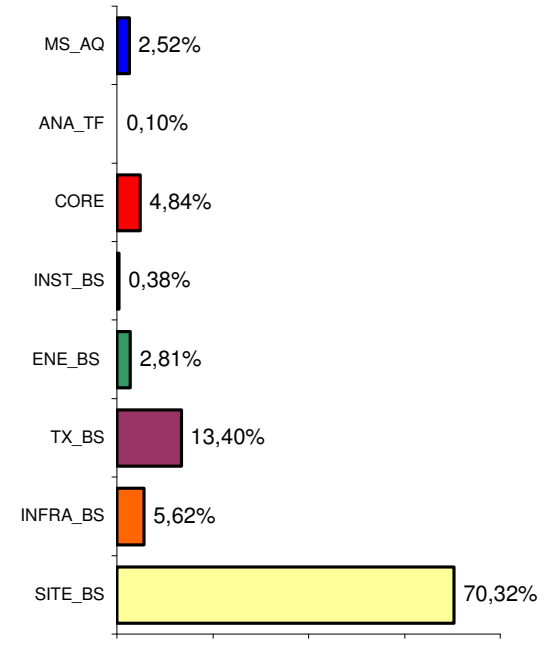

Figura 3. Capex.

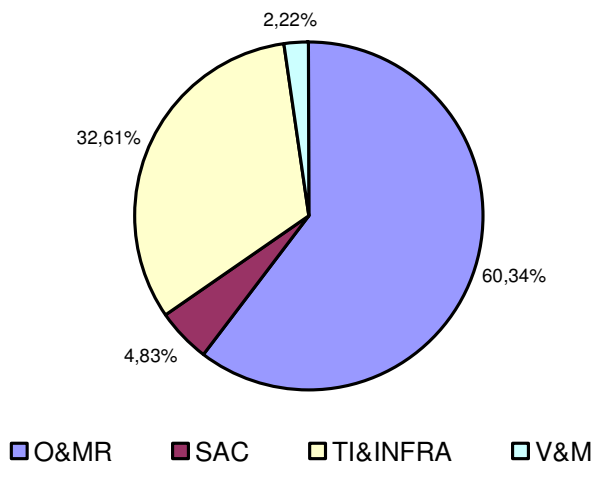

Figura 4. Opex.

\section{RESUlTADOS OBTIDOS}

Da análise, podemos perceber disparidades entre o VPL de cada um dos 3 cenários. Com a penetração do serviço baixa no Cenário 1, o Capex ficou superestimado, i.e. investimentos excessivos foram feitos para atender uma base pequena de assinantes. Neste Cenário, considerado pessimista, foi estimada a consolidação de uma base de domicílios atendidos no ano de 2016, constituída por mais de 45 mil pré-pagos e mais de 14 mil pós-pagos, totalizando $12 \%$ do total de domicílios alvo. Ao fim de 5 anos, Figura 5, as receitas não foram suficientes para cobrir as despesas, o VPL foi negativo e maior que R\$ 44 milhões, Esse resultado negativo é fruto da ineficiência empresarial do cenário. Com captação de clientes maior que no caso anterior, no Cenário 2, tanto o Capex como o Opex são suficientemente remunerados ao final do quinto ano de operação, Figura 6. O VPL foi igual a R \$ 31,8 milhões. Neste Cenário, considerado mais realista da análise, foi estimada a consolidação de uma base de domicílios atendidos no ano de
2016, constituída por 83 mil pré-pagos e 39 mil pós-pagos, que representam $24 \%$ do alvo do cenário. Já no Cenário 3, mais otimista, foi estimada uma base de domicílios atendidos com 85 mil pré-pagos e 49 mil pós-pagos, totalizando $26 \%$ do alvo e resultando num VPL igual a R\$ 84 milhões, Figura 7. Da mesma forma que no Cenário 2, se o serviço modelado no Cenário 3 for prestado com maior eficiência empresarial, i.e. com Capex e Opex proporcionais à captação de clientes, essa prestação, importante para as populações rurais que não tem acesso aos serviços de telecomunicações, torna-se ainda mais viável.

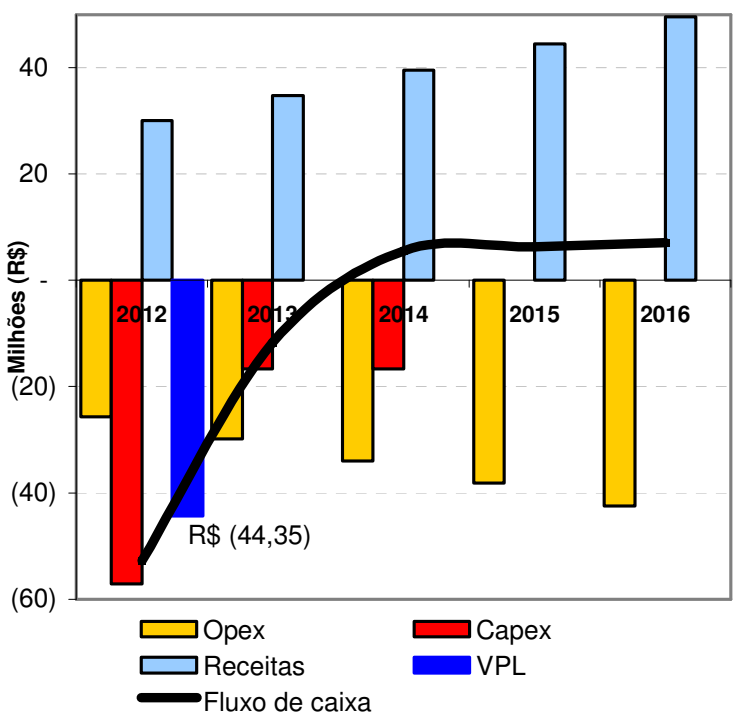

Figura 5. Resultados. Cenário 1.

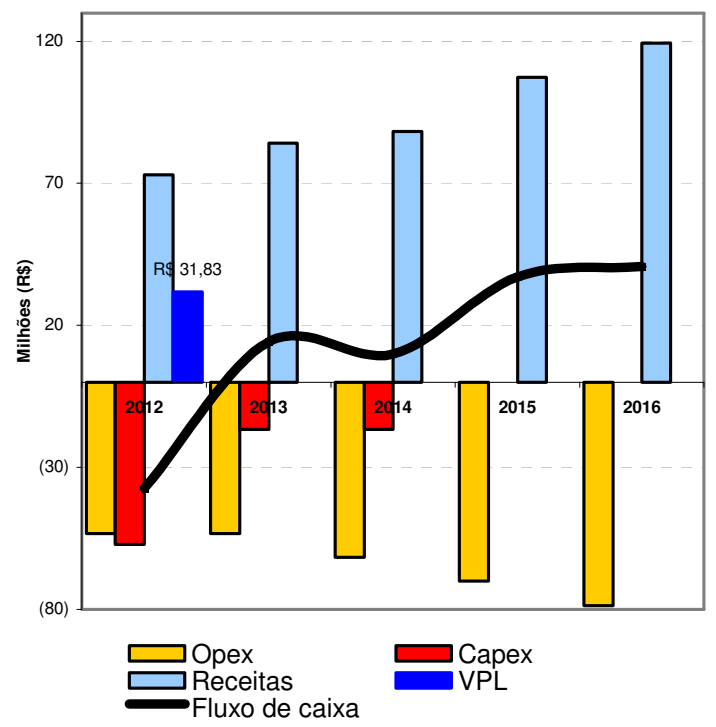

Figura 6. Resultados. Cenário 2. 


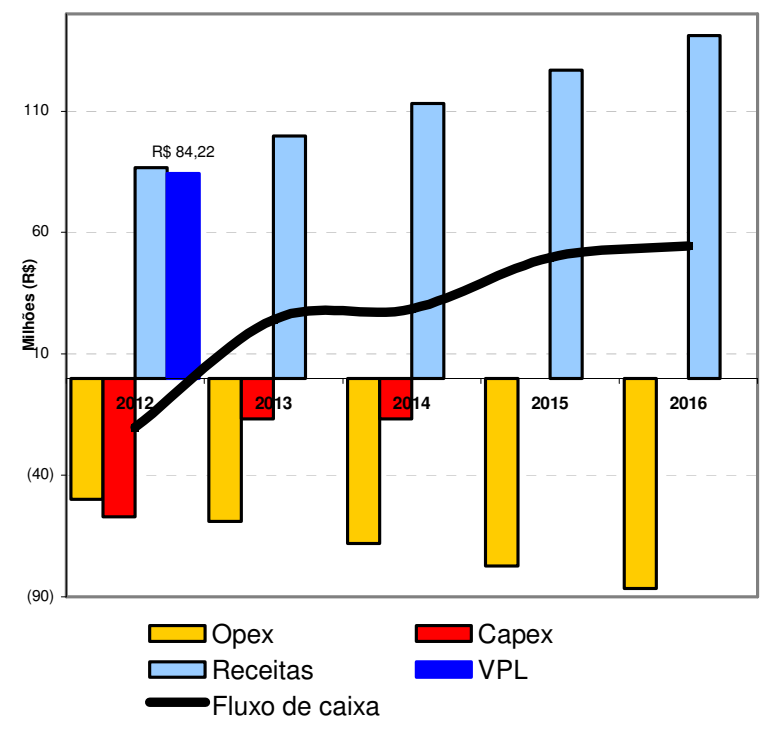

Figura 7. Resultados. Cenário 3.

\section{CONCLUSÕES}

Apresentamos uma análise tecnico-econômica da prestação do STFC em áreas rurais, por meio de um modelo de negócios inovador, baseado no uso de redes de acesso WLL, associada à tecnologia CDMA2000 sob o espectro $450 \mathrm{MHz}$, com base no índice financeiro VPL, utilizado para concluir sobre a sustentabilidade dos investimentos. Além das informações técnicas, este estudo forneceu indicadores chave para um plano de negócios como Capex, Opex, receitas, ARPU, VPL (para um dado WACC) e fluxo de caixa. Diversas conclusões podem ser depreendidas dos resultados. A primeira delas é a respeito da diferença entre as tarifas das áreas urbana e rural. Considerando que neste serviço não há cobrança de valores de meios adicionais para chamadas recebidas ou terminadas, que não há disponibilidade de oferta de outros serviços nas áreas rurais e que há um custo em expandir a rede de prestação do STFC com tecnologia sem fio, pode-se concluir que há coerência na cobrança dos valores da cesta de serviços maiores que os cobrados na área urbana, em vias de garantir a auto sustentabilidade do serviço. Além disso, face à indisponibilidade do SMP em determinadas regiões, os custos com o serviço ora desenhado, mesmo sendo em termos numéricos mais elevados do que os custos da prestação em área urbana, o tornam atrativo para os residentes em áreas rurais, além de ser um produto substitutivo à oferta de serviços limitados em qualidade e disponibilização de funcionalidades, como o antigo Ruralcel. A segunda conclusão diz respeito ao Capex e ao Opex considerados nas análises. Os preços dos produtos componentes dessas cestas dependem de diversos fatores inerentes à prestação, como a eficiência empresarial, bem como dependem de fatores macro-econômicos como a escala de mercado dos equipamentos de determinada tecnologia, e.g., equipamentos CDMA, em especial os terminais de usuários, não tem no Brasil a mesma escala que tem em países como os Estados Unidos. Os equipamentos de rede chegam a ter custos $30-50 \%$ maiores do que os equipamentos de tecnologia GSM. Os resultados positivos obtidos apontam uma tendência de que, se prestado com eficiência superior à apresentada neste trabalho, o STFC rural é um negócio rentável. Frise-se que a subestimação do Capex/Opex, bem como majorações das receitas podem alterar os resultados do projeto como um todo. O objetivo deste trabalho não foi esgotar o planejamento de uma rede WLL CDMA450, mas, traduzir em cenários hipotéticos, os resultados de uma empresa que atue sobre a observância dos parâmetros considerados. Todo modelo de negócios deve ser baseado em minuciosa análise tecnico-econômica que possa fornecer expectativas confiáveis do comportamento financeiro e da sustentabilidade dos investimentos.

\section{AGRADECIMENTOS}

O autor agradece aos colegas da Superintendência de Serviços Públicos da Anatel pelo suporte na construção do plano de negócios e nas análises econômicas desempenhadas.

\section{REFERÊNCIAS}

[1] R. Criscolo, "Rural Telecommunications in Latin América", IEEE Transactions on Communications, v. 24, pp. 325-329, Março 1976.

[2] (2009) Site da Anatel. [Online]. Disponível: http://www.anatel.gov.br.

[3] H. E. Hudson, "Overcoming the barriers of distance: telecommunications and ruraldevelopment" IEEE Technology and Society Magazine, v. 8, pp.7-10, Dezembro 1989.

[4] R. Westerveld e R. Prasad, "Rural communications in India using fixed cellular radio systems", IEEE Communications Magazine, v. 32, pp. 7077, Outubro 1994.

[5] D. Tomimura e V. V. Neto, "Field Measurements of Broadband PLC: A Case Study in the Brazilian Regulation" IEEE Global Telecommunications Conference, GLOBECOM 2008, pp. 1-4, New Orleans, EUA, Novembro 2008.

[6] E. J. B. Rodrigues; G. Cavalvcante; J. C. Silva, "Capacity Analysis and Coverage Comparison for IMT-2000 Systems in Brazilian Cities" $4^{\text {th }}$ IEEE International Conference on Wireless Communications, Networking and Mobile Computing, WICOM '08. pp. 1-4, Dalian, China, Outubro 2008.

[7] Tanveer-ul-Haq et al., "Wireless Local Loop and the Limited Mobility Issue in Pakistan" First International Conference on Information and Communication Technologies, ICICT 2005. pp. 313-317, Agosto 2005.

[8] R. Ahmad et al., "CDMA Based WLL Network Deployment Issues and Guidelines for Developing Countries", $6^{\text {th }}$ IEE International Conference on $3 \mathrm{G}$ and Beyond, Washington, EUA, pp. 1-5, Novembro 2005.

[9] (2009) Site do CDG. [Online]. Disponível: http://www.cdg.org.

[10] Y. Mahmood, "Feasibility of CDMA Technology for Providing WLL Services in Pakistan", Student Conference on Engineering Sciences and Technology, SCONEST 2005, v.27, pp. 1-6, Agosto 2005.

[11] S. Nedevschi et al., "Potential of CDMA450 for Rural Network Connectivity", IEEE Communications Magazine, Janeiro 2007.

[12] Instituto Brasileiro de Geografia e Estatística (IBGE), Pesquisa Nacional por Amostra de Domicílios (PNAD), 2007.

[13] Instituto Brasileiro de Geografia e Estatística (IBGE), Pesquisa de Orçamento Familiar (POF), 2003.

[14] B. T. Olsen et al. "Technoeconomic evaluation of the major telecommunication investment options for European players" IEEE Network, v.20, pp. 6-15, Julho 2006.

[15] S. Mishra et al. "Economic Analysis of Networking Technologies for Rural Developing Regions" 1st International Workshop on Internet and Network Economics, WINE 2005, Hong Kong, China, Dezembro 2005. 